Article

\title{
Design and Evaluation of Novel Polymyxin Fluorescent Probes
}

\author{
Bo Yun ${ }^{1}$, Kade D. Roberts ${ }^{1}$, Philip E. Thompson ${ }^{2}$, Roger L. Nation ${ }^{1}$, Tony Velkov ${ }^{1, *}$ and \\ Jian $\mathrm{Li}^{3, *}$ \\ 1 Drug Delivery, Disposition and Dynamics, Monash University, Parkville, Victoria 3052, Australia; \\ Bo.Yun@petermac.org (B.Y.); Kade.Roberts@monash.edu (K.D.R.); roger.nation@monash.edu (R.L.N.) \\ 2 Medicinal Chemistry, Monash Institute of Pharmaceutical Sciences, Monash University, Parkville, \\ Victoria 3052, Australia; Philip.Thompson@monash.edu \\ 3 Monash Biomedicine Discovery Institute, Department of Microbiology, Monash University, Clayton, \\ Victoria 3800, Australia \\ * Correspondence: tony.velkov@unimelb.edu.au (T.V.); jian.li@monash.edu (J.L.)
}

Received: 12 October 2017; Accepted: 9 November 2017; Published: 11 November 2017

\begin{abstract}
Polymyxins (polymyxin B and colistin) are cyclic lipopeptide antibiotics that serve as a last-line defence against Gram-negative "superbugs". In the present study, two novel fluorescent polymyxin probes were designed through regio-selective modifications of the polymyxin B core structure at the $\mathrm{N}$-terminus and the hydrophobic motif at positions 6 and 7 . The resulting probes, FADDI-285 and FADDI-286 demonstrated comparable antibacterial activity (MICs $2-8 \mathrm{mg} / \mathrm{L}$ ) to polymyxin B and colistin (MICs $0.5-8 \mathrm{mg} / \mathrm{L}$ ) against a panel of gram-negative clinical isolates of Acinetobacter baumannii, Klebsiella pneumoniae and Pseudomonas aeruginosa. These probes should prove to be of considerable utility for imaging cellular uptake and mechanistic investigations of these important last-line antibiotics.
\end{abstract}

Keywords: polymyxins; fluorescent; dansyl; probe; gram-negative

\section{Introduction}

Over the past two decades there has been a pronounced increase in the emergence of multidrug-resistant (MDR) Gram-negative "superbugs", leading to serious infections that are resistant to almost all currently available antibiotics [1]. The dire situation is perpetuated by a lack of novel antibiotics in the developmental pipeline, leaving the world in a vulnerable state against these life-threatening infections [1]. This "perfect storm" has led to the revival of the polymyxin class of antibiotics, polymyxin B and E (the latter also known as colistin), as a last line of defence against MDR Gram-negative "superbugs" [2]. However, despite their excellent antibacterial activity, the use of polymyxins has largely been limited by a high incidence of nephrotoxicity among patients receiving these antibiotics [3-6].

Polymyxins are amphipathic cationic lipopeptides, comprised of hydrophobic and hydrophilic domains that are critical for their antibacterial activity [7]. The general polymyxin structure consists of a cyclic heptapeptide ring with a linear tripeptide segment and an $N$-terminal fatty acyl tail (Figure 1). Additionally, there are five L- $\alpha, \gamma$-diaminobutyric acid (Dab) residues, which contain primary amines that are positively charged at physiological $\mathrm{pH}(7.4)$, as well as two hydrophobic residues in positions 6 and 7 of the cyclic ring. The two polymyxins used clinically, polymyxin B and colistin, are differentiated by a single hydrophobic residue at position 6: D-leucine in colistin and D-phenylalanine in polymyxin $B$ [7]. Both polymyxins are products of fermentation and are mixtures, each containing two major components, colistin $A$ and $B$ and polymyxin $B_{1}$ and $B_{2}$, which differ by one carbon at the fatty acyl tail (Figure 1). The fatty acyl tail is essential for the antibacterial activity 
of polymyxins, since polymyxin nonapeptide (PMBN) (produced by proteolytic removal of the fatty acyl-Dab1 from the $N$-terminus of the polymyxin) is inactive [8-10]. These structural features of the polymyxin core scaffold are critical for interaction with the initial target, lipid A of the outer membrane.

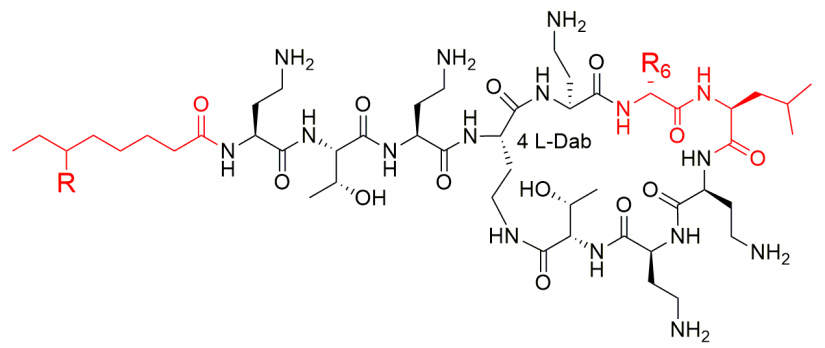

\footnotetext{
Polymyxin $\mathrm{B}_{1} \mathrm{R}=\mathrm{CH}_{3}, \mathrm{R}_{6}=\mathrm{D}$-Phe Colistin $\mathrm{A} \mathrm{R}=\mathrm{CH}_{3}, \mathrm{R}_{6}=\mathrm{D}$-Leu

Polymyxin $B_{2} R=H, R_{6}=D$-Phe Colsitin $B R=H, R_{6}=D$-Leu
}
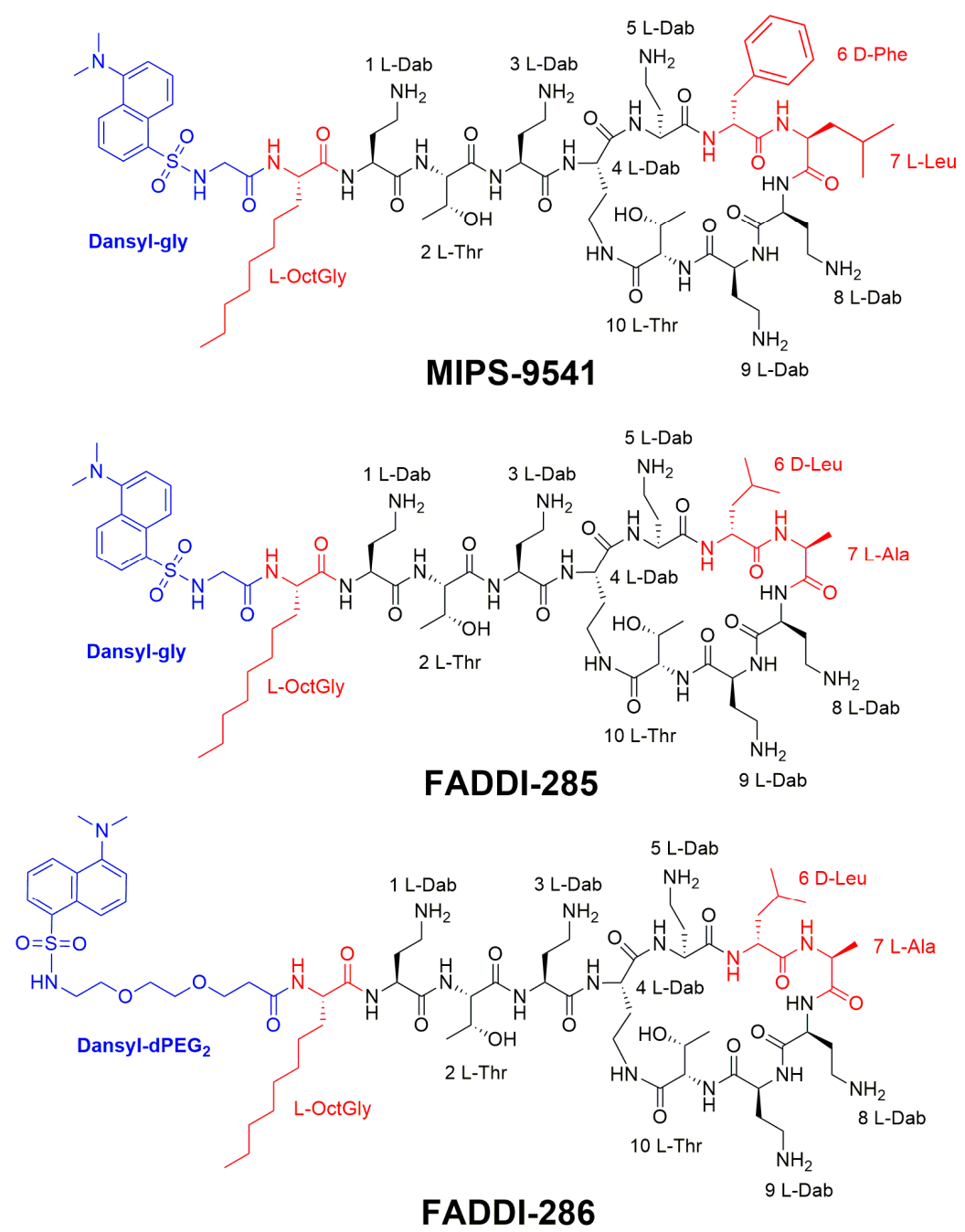

Figure 1. Structures of polymyxin B, colistin, MIPS-9541 and the novel fluorescent polymyxin probes FADDI-285 and FADDI-286.

Commercial probes (e.g., dansyl- and BODIPY-polymyxin B) have been utilized in polymyxin mechanistic studies, however, they lack antimicrobial activity due to the blockage of multiple Dab residues (potentially up to all five); therefore, these compounds are not structurally representative 
of the parent polymyxin [11]. Our group has previously reported the design and synthesis of regio-selectively mono-dansylated polymyxin B probes such as MIPS-9541 (Figure 1) for exploring polymyxin mechanisms of action and imaging of polymyxin interactions with kidney proximal tubular cells [12]. In the current study, we build on our novel design strategy generating the novel fluorescently labelled polymyxin probes, FADDI-285 and FADDI-286 (Figure 1) which are representative of the native polymyxins, and retain antimicrobial activity. These novel fluorescent polymyxin probes should have improved in vivo utility and help facilitate medicinal chemistry strategies to ameliorate unwanted nephrotoxicity and resistance that limit the clinical efficacy of these important last-line lipopeptide antibiotics.

\section{Methods}

\subsection{Chemical Reagents}

Diisopropylethylamine (DIPEA) was obtained from Auspep (Melbourne, Australia). Fmoc-L-OctGly-OH and Fmoc-Dab(Boc)-OH were obtained from Try-lead Chem (Hangzhou, China). Fmoc-Dab(ivDde)-OH, Fmoc-D-Leu-OH, 1H-Benzotriazolium-1-[bis(dimethylamino)methylene]-5-chloro hexafluoro- phosphate-(1-),3-oxide (HCTU) and 1,1,1,3,3,3-Hexafluoro-2-propanol (HFIP) were obtained from Chem-Impex International (Wood Dale, IL, USA). Fmoc-Thr(tBu)-OH, Fmoc-Ala-OH and Fmoc-Gly-OH were obtained from Mimotopes (Melbourne, Australia). N-Fmoc-Amido-dPEG $-\mathrm{OH}$ was obtained from Peptides International (Louisville, KY, USA). Dichloromethane (DCM), dimethylformamide (DMF), diethyl ether and acetonitrile were obtained from Merck (Melbourne, Australia). Fmoc-Thr(tBu)-TCP-Resin was obtained from Intavis Bioanyltical Instruments (Köln, Germany). Piperidine, triisopropylsilane (TIPS), trifluoroacetic acid (TFA), dansyl-chloride, ethanedithiol (EDT) and diphenylphosphorylazide (DPPA) were obtained from Sigma-Aldrich (Castle Hill, Australia) Polymyxin B sulfate and colistin sulfate were research grade and obtained from BetaPharma (Shanghai, China).

\subsection{HPLC Purification and LC-MS Analysis}

Peptides were purified by RP-HPLC on a Waters Prep LC system incorporating a Waters 486 tuneable absorbance detector set at $214 \mathrm{~nm}$ and a Phenomenex Luna C8(2) column $(250 \times 21.2 \mathrm{~mm} \mathrm{ID}$, $100 \AA, 10$ micron). Peptides were eluted with a gradient of $100 \%$ Buffer A $(0.1 \%$ TFA/water $)$ to $60 \%$ Buffer B (0.1\%TFA/acetonitrile) over $60 \mathrm{~min}$ at a flow rate of $15 \mathrm{~mL} / \mathrm{min}$. Fractions collected were analysed by LC/MS on a Shimadzu 2020 LCMS system. LC analysis was carried out at $214 \mathrm{~nm}$ using a Phenomenex Luna C8(2) column $(100 \times 2.0 \mathrm{~mm}$ ID, $100 \AA$, 3 micron $)$, eluting with a gradient of $100 \%$ Buffer A (0.05\% TFA/water) to $60 \%$ Buffer B (0.05\%TFA/acetonitrile) over $10 \mathrm{~min}$ at a flow rate of $0.2 \mathrm{~mL} / \mathrm{min}$. Mass spectra were acquired in positive ion mode with a scan range of $200-2000 \mathrm{~m} / \mathrm{z}$.

\subsection{Synthesis FADDI-285}

Synthesis of the protected linear peptide precursor was conducted on a Protein Technologies Prelude automated peptide synthesizer using pre-loaded Fmoc-Thr $(\mathrm{tBu})-\mathrm{TCP}$ resin $(0.1 \mathrm{mmol}$ scale). Fmoc deprotection was conducted using $20 \%$ piperidine in dimethylformamide $(1 \times 5 \mathrm{~min}, 1 \times 10 \mathrm{~min})$ at room temperature. Coupling of the Fmoc-amino acids for $50 \mathrm{~min}$ at room temperature using 3 molar equivalents of the Fmoc-amino acid and HCTU in DMF activated in situ, using 6 molar equivalents of DIPEA. The $N$-terminal dansyl group was coupled using 3 molar equivalents of dansyl-chloride in DMF in the presence of 6 molar equivalents of DIPEA for $50 \mathrm{~min}$ at room temperature. The resin was then treated with $3 \%$ Hydrazine/ DMF $(4 \times 15 \mathrm{~min})$ to remove the ivDde group. The protected linear peptide was then cleaved from the resin by treating the resin with $10 \%$ HFIP in DCM $(1 \times 30 \mathrm{~min}$, $1 \times 5 \mathrm{~min}$ ). This solution was concentrated in vacuo to produce the protected linear peptide as a residue. The protected linear peptide was dissolved in DMF (10 mL) to which DPPA $0.3 \mathrm{mmol}$, $0.65 \mu \mathrm{L}$ (3 molar equivalents relative to the loading of the resin) and DIPEA $0.6 \mathrm{mmol}, 104 \mu \mathrm{L}$ (6 molar 
equivalents relative to the loading of the resin) were added. This solution was stirred at room temperature overnight. The reaction solution was then concentrated under vacuum overnight to give the crude protected cyclic peptide as a residue. The resulting residue was taken up in a solution of $2.5 \%$ EDT $/ 5 \%$ TIPS/TFA and shaken at room temperature for $2 \mathrm{~h}$. To this solution $40 \mathrm{~mL}$ of diethyl ether was added. The resulting precipitate was collected by centrifugation and washed twice more with diethyl ether $(40 \mathrm{~mL})$ then air-dried in a fume hood to give the crude cyclic peptide as a solid. The resulting solid was taken up in Milli-Q water $(5 \mathrm{~mL})$ and de-salted using a Vari-Pure IPE SAX column. The eluent containing the crude cyclic peptide was acidified with TFA (10 L) and subjected to RP-HPLC purification as described above. Fractions collected were analysed by LC-MS as described above. Fractions containing the desired product were freeze-dried to give the FADDI-285 TFA salt as a pale-yellow solid in a yield of $57.2 \mathrm{mg}$ ( $>95 \%$ purity). Molecular weight was confirmed by ESI-MS analysis; $\mathrm{m} / \mathrm{z}$ (monoisotopic) calculated: $\mathrm{C}_{65} \mathrm{H}_{111} \mathrm{~N}_{19} \mathrm{O}_{16} \mathrm{~S} 1446.82$, $[\mathrm{M}+2 \mathrm{H}]^{2+} 723.91$, $[\mathrm{M}+3 \mathrm{H}]^{3+}$ 482.93; observed: $[\mathrm{M}+2 \mathrm{H}]^{2+}$ 724.30. $[\mathrm{M}+3 \mathrm{H}]^{3+} 483.50$.

\subsection{Synthesis FADDI-286}

This peptide was synthesized as described above for FADDI-285 to give the FADDI-286 TFA salt as a pale-yellow solid in a yield of $65.2 \mathrm{mg}$ ( $>95 \%$ purity). Molecular weight was confirmed by ESI-MS analysis; $\mathrm{m} / \mathrm{z}$ (monoisotopic) calculated: $\mathrm{C}_{70} \mathrm{H}_{121} \mathrm{~N}_{19} \mathrm{O}_{18} \mathrm{~S}[\mathrm{M}+\mathrm{H}]^{+} 1548.89,[\mathrm{M}+2 \mathrm{H}]^{2+} 774.94$, $[\mathrm{M}+3 \mathrm{H}]^{3+}$ 516.96; observed: $[\mathrm{M}+2 \mathrm{H}]^{2+} 775.40,[\mathrm{M}+3 \mathrm{H}]^{3+} 517.60$.

\subsection{Determination of MICs}

MICs against Pseudomonas aeruginosa, Klebsiella pneumoniae, and Acinetobacter baumannii strains were determined by the broth microdilution method (CLSI 2013). Experiments were conducted in 96-well polypropylene microtitre plates with all dilutions using cation-adjusted Mueller-Hinton broth (CaMHB). Bacterial suspension (100 $\mu \mathrm{L}$, containing $\sim 10^{6}$ colony forming units (CFU) per $\mathrm{mL}$ ) was added to the wells in the presence of increasing concentrations of polymyxins ( 0 to $128 \mathrm{mg} / \mathrm{L}$ ). MICs are defined as the lowest concentration at which visible growth was inhibited after overnight incubation at $37^{\circ} \mathrm{C}$.

\section{Results}

\subsection{Probe Design and Synthesis}

Previously we designed the regio-selectively mono-dansylated probe MIPS-9541 in which the $\mathrm{N}$-terminal fatty acyl group of polymyxin B was substituted with dansylglycine-octanylglycine (Figure 1) [11]. The hydrophobic dansyl group was utilized as the fluorophore, as its comparatively small size relative to other fluorophores would help to reduce the likelihood of negative steric effects on the polymyxin pharmacophore [7]. The L-octylglycine (C8) residue serves to emulate the eight carbon $N$-terminal fatty acyl chain of the polymyxins and also provides a point for attachment of the dansyl fluorophore [11]. It has been previously demonstrated that a variety of hydrophobic groups are well tolerated at this position and act as mimics of the $N$-terminal saturated alkyl fatty acyl chains of polymyxin B [7]. NMR analysis showed that this probe has a similar mode of binding to lipid A, the initial target of the polymyxins such as the native polymyxin B (Figure 2). One of the concerns with the design of MIPS-9541, was the increase in the overall hydrophobicity of the scaffold resulting from the addition of the dansyl fluorophore which may have a negative effect on the ability of the molecule to closely mimic the native polymyxins. For example, increased hydrophobicity could lead to increased plasma protein binding leading to poor bio-distribution compared to the native polymyxins, which would have a negative impact on in vivo studies utilizing the probe [13]. In the current work, we have made further modification to MIPS-9541 in order to balance the hydrophobicity of the scaffold and improve its in vivo utility. To this end we incorporated a D-leucine residue at position 6 in place of D-Phe as seen in colistin and reduced the hydrophobicity at position 7 by substituting the 
leucine residue with a less hydrophobic alanine residue. This resulted in the generation of FADDI-285 (Figure 1). Previously it had been shown that the leucine residue at position 7 could be substituted with an alanine residue without loss of antibacterial activity against $P$. aeruginosa [14]. Further to these modifications, the glycine linker between the dansyl fluorophre and octylglycine residue was replaced with a PEG linker to generate FADDI-286. This PEG linker would help to decrease the hydrophobicity of the dansyl-octylglycine $N$-terminal modification.

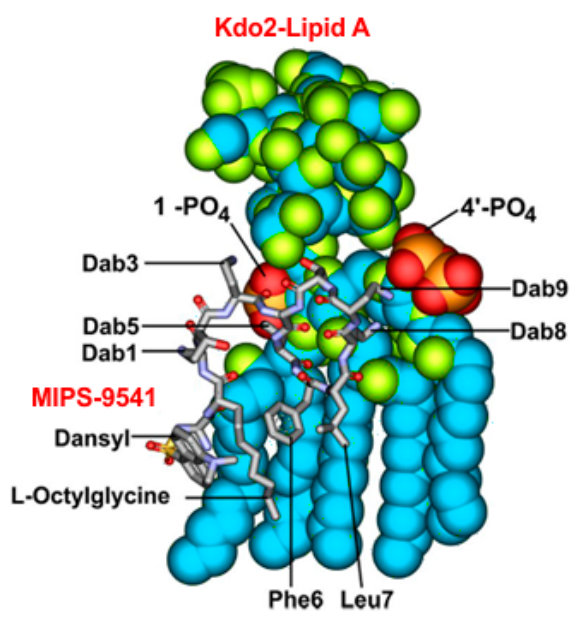

Figure 2. NMR derived model of MIPS-9541 in complex with bacterial Kdo2-lipid.

FADDI-285 and FADDI-286 had to be prepared using a total synthesis approach and were readily synthesized using standard solid-phase peptide synthesis and commercially available chemical reagents. Pleasingly, the modifications made to the polymyxin structure did not have a negative impact on the key cyclisation step to generate the heptapeptide cyclic ring, with final products being obtained in good yield and purity.

\subsection{Antibacterial Activity of the Probes}

Antibacterial activity of both probes was assessed against the American Type Culture Collection (ATCC) strains and a panel of clinical isolates of polymyxin-susceptible P. aeruginosa, K. pneumoniae and A. baumannii (Table 1). Both probes demonstrated antibacterial activity against the polymyxin-susceptible strains (MICs $2-8 \mathrm{mg} / \mathrm{L}$ ), comparable to polymyxin B and colistin (MICs 0.5-8 mg/L). Notably, both probes exhibited enhanced activity (MICs $\sim 32 \mathrm{mg} / \mathrm{L}$ ) against the polymyxin-resistant isolates (polymyxin B and colistin MICs $~ 128 \mathrm{mg} / \mathrm{L}$ ). 
Table 1. Minimum inhibitory concentrations (MICs) of each dansylated probe, polymyxin B and colistin against Gram-negative bacteria.

\begin{tabular}{|c|c|c|c|c|c|c|c|c|c|c|c|c|c|c|c|c|}
\hline & 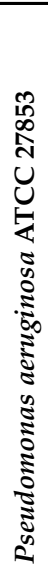 & 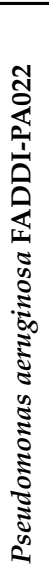 & 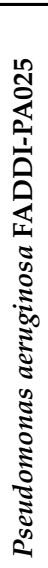 & 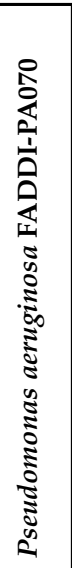 & 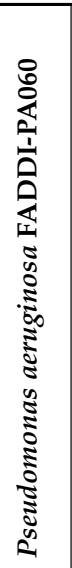 & 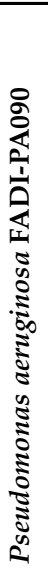 & 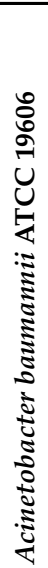 & 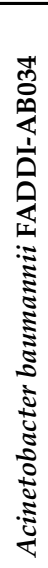 & 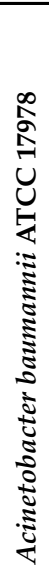 & 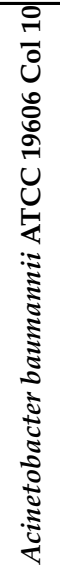 & 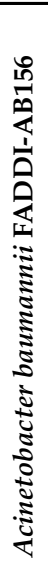 & 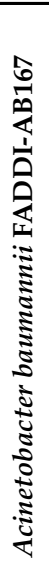 & 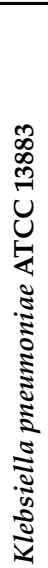 & 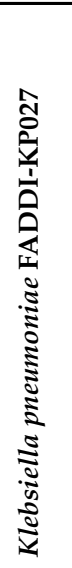 & 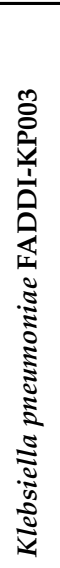 & 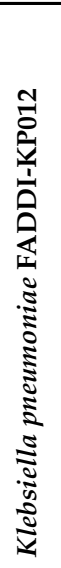 \\
\hline Peptide & \multicolumn{16}{|c|}{ MIC $(\mathrm{mg} / \mathrm{L})$} \\
\hline Colistin & 1 & 1 & 2 & $>128$ & $>128$ & 8 & 1 & 0.5 & 0.5 & 128 & 16 & 8 & 1 & $>128$ & 128 & 32 \\
\hline PolymyxinB & 1 & 1 & 1 & 32 & $>32$ & 4 & 1 & 0.5 & 1 & 128 & 8 & 16 & 1 & 128 & $>32$ & 16 \\
\hline FADDI-285 & 2 & 2 & 4 & $>32$ & 2 & 8 & 4 & 4 & 8 & $>32$ & 8 & 8 & 4 & $>32$ & $>32$ & $>32$ \\
\hline FADDI-286 & 2 & 2 & 4 & $>32$ & 2 & 4 & 4 & 4 & 4 & $>32$ & 8 & 8 & 4 & $>32$ & $>32$ & $>32$ \\
\hline
\end{tabular}

\section{Discussion and Conclusions}

While bacteria have developed resistance to almost all other antibiotics, colistin and polymyxin B remain at the forefront of last-line therapeutics against MDR Gram-negative "superbugs" [15]. The primary focus of this study was to design and synthesize novel polymyxin fluorescent probes with the antibacterial activity representative of the native polymyxins.

Polymyxins elicit their antimicrobial activity by first binding to the lipopolysaccharide in the bacterial outer membrane. The formation of the complex is initiated through the electrostatic interaction of the cationic Dab side-chains with the negatively charged phosphate groups of the lipid a component of LPS. This in turn displaces divalent cations $\left(\mathrm{Ca}^{2+}\right.$ and $\left.\mathrm{Mg}^{2+}\right)$ that bridge adjacent LPS molecules, thereby de-stabilizing the outer membrane $[2,7,16]$. Subsequently, hydrophobic interactions occur between the $N$-terminal fatty acyl tail and the positions 6 and 7 hydrophobic segment of the polymyxin molecule and the fatty acyl chains of lipid A. The physical integrity of the phospholipid bilayer in the inner membrane appears to be subsequently disrupted [16-20]. This 'self-promoted' uptake mechanism is believed to lead to disruption of the cell envelope and bacterial killing $[7,17,18,21]$. With these principles in mind, we rationally designed the probes FADDI-285 and FADDI-286 via regio-selective modifications at the hydrophobic $\mathrm{N}$-terminus and position 7 , without markedly compromising antimicrobial activity of the parent colistin compound. Our group has previously highlighted the pitfalls of directly coupling fluorescent groups such as dansyl directly onto the Dab side chains in semi-synthetic preparations of dansyl-polymyxin B [11]. Furthermore, as polymyxin $\mathrm{B}$ and colistin are each comprised of two major components (polymyxin $\mathrm{B}_{1}$ and $\mathrm{B}_{2}$; colistin $\mathrm{A}$ and B), there is a strong possibility that either of these components will be substituted at any of the five Dab side chains resulting in a highly heterogeneous mixture of dansylated derivatives [11]. Indeed our previously reported mass spectrometry analysis of these semi-synthetic dansyl-polymyxin $\mathrm{B}$ preparations indicated the presence of a heterogeneous mixture of mono-, di-, and tri-dansyl Dab-substituted polymyxin B [11]. Accordingly, there is little value in using these semi-synthetic preparations as probes for imaging localization, since they lack the native antibacterial activity of the polymyxin B parent molecule and resultant images would represent the localization of a very complex array of probe molecules. Both FADDI-285 and FADDI-286 possess antibacterial activity similar to colistin and polymyxin B against polymyxin-susceptible isolates and notably, 4-fold enhanced activity against the polymyxin-resistant isolates (Table 1), and as such should prove to be of considerable utility 
as tools for novel polymyxin lipopeptide discovery programs. The utility of these probes in vivo is being investigated and will be the subject of a future report.

Acknowledgments: J.L. is an Australian NHMRC Senior Research Fellow. T.V. is an Australian NHMRC Industry Career Development Research Fellow.

Author Contributions: B.Y., K.D.R. performed the experiments and helped write the manuscript. R.L.N. and P.T. helped write the manuscript. K.D.R., T.V. and J.L. designed the experiments and wrote the manuscript.

Conflicts of Interest: The authors declare no conflict of interest.

\section{References}

1. Boucher, H.W.; Talbot, G.H.; Benjamin, D.K., Jr.; Bradley, J.; Guidos, R.J.; Jones, R.N.; Murray, B.E.; Bonomo, R.A.; Gilbert, D. $10 \times$ '20 Progress-Development of New Drugs Active Against Gram-Negative Bacilli: An Update From the Infectious Diseases Society of America. Clin. Infect. Dis. 2013, 56, 1685-1694. [CrossRef] [PubMed]

2. Velkov, T.; Roberts, K.D.; Nation, R.L.; Thompson, P.E.; Li, J. Pharmacology of polymyxins: New insights into an "old" class of antibiotics. Future Microbiol. 2013, 8, 711-724. [CrossRef] [PubMed]

3. Landman, D.; Georgescu, C.; Martin, D.A.; Quale, J. Polymyxins revisited. Clin. Microbiol. Rev. 2008, 21, 449-465. [CrossRef] [PubMed]

4. Akajagbor, D.S.; Wilson, S.L.; Shere-Wolfe, K.D.; Dakum, P.; Charurat, M.E.; Gilliam, B.L. Higher Incidence of Acute Kidney Injury With Intravenous Colistimethate Sodium Compared with Polymyxin B in Critically Ill Patients at a Tertiary Care Medical Center. Clin. Infect. Dis. 2013, 57, 1300-1303. [CrossRef] [PubMed]

5. Hartzell, J.D.; Neff, R.; Ake, J.; Howard, R.; Olson, S.; Paolino, K.; Vishnepolsky, M.; Weintrob, A.; Wortmann, G. Nephrotoxicity Associated with Intravenous Colistin (Colistimethate Sodium) Treatment at a Tertiary Care Medical Center. Clin. Infect. Dis. 2009, 48, 1724-1728. [CrossRef] [PubMed]

6. Kubin, C.J.; Ellman, T.M.; Phadke, V.; Haynes, L.J.; Calfee, D.P.; Yin, M.T. Incidence and predictors of acute kidney injury associated with intravenous polymyxin B therapy. J. Infect. 2012, 65, 80-87. [CrossRef] [PubMed]

7. Velkov, T.; Thompson, P.E.; Nation, R.L.; Li, J. Structure-activity relationships of polymyxin antibiotics. J. Med. Microbiol. 2010, 53, 1898-1916. [CrossRef] [PubMed]

8. Ofek, I.; Cohen, S.; Rahmani, R.; Kabha, K.; Tamarkin, D.; Herzig, Y.; Rubinstein, E. Antibacterial synergism of polymyxin $\mathrm{B}$ nonapeptide and hydrophobic antibiotics in experimental gram-negative infections in mice. Antimicrob. Agents Chemother. 1994, 38, 374-377. [CrossRef] [PubMed]

9. Tsubery, H.; Ofek, I.; Cohen, S.; Fridkin, M. Structure-activity relationship study of polymyxin B nonapeptide. Adv. Exp. Med. Biol. 2000, 479, 219-222. [PubMed]

10. Tsubery, H.; Ofek, I.; Cohen, S.; Fridkin, M. N-terminal modifications of polymyxin B nonapeptide and their effect on antibacterial activity. Peptides 2001, 22, 1675-1681. [CrossRef]

11. Deris, Z.Z.; Swarbrick, J.D.; Roberts, K.D.; Azad, M.A.K.; Akter, J.; Horne, A.S.; Nation, R.L.; Rogers, K.L.; Thompson, P.E.; Velkov, T.; Li, J. Probing the Penetration of Antimicrobial Polymyxin Lipopeptides into Gram-Negative Bacteria. Bioconj. Chem. 2014, 25, 750-760. [CrossRef] [PubMed]

12. Yun, B.; Azad, M.A.K.; Nowell, C.J.; Nation, R.L.; Thompson, P.E.; Roberts, K.D.; Velkov, T.; Li, J. Cellular Uptake and Localization of Polymyxins in Renal Tubular Cells Using Rationally Designed Fluorescent Probes. Antimicrob. Agents Chemother. 2015, 59, 7489-7496. [CrossRef] [PubMed]

13. Velkov, T.; Roberts, K.D.; Nation, R.L.; Wang, J.; Thompson, P.E.; Li, J. Teaching 'Old' Polymyxins New Tricks: New-Generation Lipopeptides Targeting Gram-Negative 'Superbugs'. ACS Chem. Biol. 2014, 9, 1172-1177. [CrossRef] [PubMed]

14. Kanazawa, K.; Sato, Y.; Ohki, K.; Okimura, K.; Uchida, Y.; Shindo, M.; Sakura, N. Contribution of Each Amino Acid Residue in Polymyxin B3 to Antimicrobial and Lipopolysaccharide Binding Activity. Chem. Pharm. Bull. 2009, 57, 240-244. [CrossRef] [PubMed]

15. Nation, R.L.; Velkov, T.; Li, J. Colistin and Polymyxin B: Peas in a Pod, or Chalk and Cheese? Clin. Infect. Dis. 2014, 59, 88-94. [CrossRef] [PubMed]

16. Pristovsek, P.; Kidric, J. The search for molecular determinants of LPS inhibition by proteins and peptides. Curr. Top. Med. Chem. 2004, 4, 1185-1201. [CrossRef] [PubMed] 
17. Hancock, R. The bacterial outer membrane as a drug barrier. Trends Microbiol. 1997, 5, 37-42. [CrossRef]

18. Hancock, R.E.; Lehrer, R. Cationic peptides: A new source of antibiotics. Trends Biotechnol. 1998, 16, 82-88. [CrossRef]

19. Clausell, A.; Garcia-Subirats, M.; Pujol, M.; Busquets, M.A.; Rabanal, F.; Cajal, Y. Gram-negative outer and inner membrane models: Insertion of cyclic cationic lipopeptides. J. Phys. Chem. B 2007, 111, 551-563. [CrossRef] [PubMed]

20. Powers, J.P.; Hancock, R.E. The relationship between peptide structure and antibacterial activity. Peptides 2003, 24, 1681-1691. [CrossRef] [PubMed]

21. Hancock, R.E. Peptide antibiotics. Lancet 1997, 349, 418-422. [CrossRef]

(C) 2017 by the authors. Licensee MDPI, Basel, Switzerland. This article is an open access article distributed under the terms and conditions of the Creative Commons Attribution (CC BY) license (http://creativecommons.org/licenses/by/4.0/). 